\title{
Downlink Capacity Analysis for DS-CDMA Satellite Systems Accounting for On-Board Power Constraints
}

\author{
Ernestina Cianca*, Silvia De Fina*, Roberto Lojacono* ${ }^{*}$,Marina Ruggieri* ${ }^{*}$ Ramjee Prasad ${ }^{* *}$ \\ (*) University of Roma “Tor Vergata”, Dpt. of Electronics Engineering \\ Via di Tor Vergata 110, I-00133 Roma (Italy) \\ (**) Center for PersonKommuniKation, Aalborg University (Denmark) \\ E-mail: ruggieri@uniroma2.it, cianca@nausicaa.eln.uniroma2.it
}

\begin{abstract}
This paper presents a novel methodology for the analysis of the downlink of a DS-CDMA mobile satellite system which allows to evaluate the impact of the power limitations expected in a satellite-based access in terms of capacity. The methodology has been applied to compare an optimum power control, providing BER-driven thresholds, with a pure Signal-toInterference ratio (SIR)-based strategy. Relationships among service BER, channel states statistic and expected dynamic range of the outer-loop process are derived. Concluding remarks on power management policy, and its impact on the overall capacity, are given.
\end{abstract}

\section{Introduction}

The third generation Universal Mobile Telecommunications Systems (UMTS) foresee the integration of a Terrestrial component (T-UMTS) and a Satellite component (SUMTS) in order to provide global mobility. Wide-Band Code Division Multiple Access (WCDMA) has been chosen as the basic radio access technology for the terrestrial component of UMTS/IMT-2000 (International Mobile Telecommunications-2000), [1],[2]. CDMA technology is considered for the S-UMTS component as well, in order to ease dual-mode integration (satellite/terrestrial) and to lower cost and complexity.

In the literature, most of the performance analysis of Direct Sequence (DS) CDMA mobile systems deals with the uplink that is the most critical link in terms of capacity in terrestrial mobile systems. In satellite systems, due to the on-board power constraints, the satellite-to-mobile link (downlink) is typically the bottleneck of the capacity [3]. So far, the downlink analysis has been developed either without constraints on the limited spacecraft power [4], or under the hypothesis of full orthogonality between the spreading sequences [5], [6], [3]. The latter actually applies only in the case of a very low satellite load, below the codebook limit (namely, less that 256 voice users for the Wideband CDMA (WCDMA) radio interface). In both cases, the actual sensitivity of capacity with respect to power management strategies, as power control and satellite diversity, cannot be fully appreciated.

In this frame, the present paper proposes a novel approach for the analysis of power control and diversity over the satellite-to-mobile link (downlink). Both for a single reception system and a dual-satellite diversity system, a BERdriven power-control and a SIR-Driven power control scheme are compared. Furthermore, an improved power management strategy for power limited system is proposed.

The paper is organized as follows. In Section II the system model is presented; Section III describes the analysis methodology. Results obtainable with the proposed approach are derived and discussed in Section IV. A proper power management strategy is described in Section V. In Section 6, the main results are summarized, conclusions and perspectives are drawn.

\section{System Model}

Let us assume that a total number of users $K_{S}$ are served by $M$ satellites in visibility. For each satellite an average load of $K$ users is uniformly distributed among $N_{b}$ spot beams and $N_{c}$ carriers. Each carrier is accessed according to a QPSK- 
based DS-CDMA. No time synchronization among the different satellite signals is assumed, so that the signals coming from different satellites generate asynchronous interference after despreading at the demodulator site. Cross polarization frequency reuse and Voice Activity Detection (VAD) are used to reduce interference. The actual orthogonality degree experienced in the downlink is assumed to be a function of the total number of users $K$ [7]. An hybrid satellite diversity scheme is assumed: Selection Diversity (SD) when the user is not shadowed with respect of one satellite at least; Rake receiver with Maximal Ratio Combining (MRC), when the user is shadowed with respect to all satellites. A BER-Driven power control is considered. It is a closed loop power control system which consists of two parts: an inner and an outer loop. The inner loop is a SIR-based power control, i.e. the receiver compares the estimated received signal to interference ratio (SIR) with a target value and commands the transmitter to increase or decrease the power accordingly. The loop is assumed to be able to compensate for large scale signal variations, but not capable to counteract the fast fading components. Hence, the received $E_{b} / N_{T}$ after power control, where $N_{T}$ includes both thermal noise and MAI (Multiple Access Interference), is still variable. Therefore, the effectiveness of the inner loop depends on the propagation conditions of the user of interest. The outer loop is needed to compensate such variability, adjusting the target SIR so that all users obtain the same performance in terms of bit error rate. Let us denote by $\mathbf{r}_{m j}(t)$ the complex envelope of the signal received by the $j$-th user from the $m$-th satellite, being satellite $i$ the service one; neglecting the data modulation component, we have

$$
\begin{array}{r}
\mathbf{r}_{m j}(t)=\mathbf{h}_{m j}(t)\left[\delta_{0}[m-i] \sqrt{2 P_{j}} \mathbf{c}_{j}\left(t-\tau_{m j}\right)+\right. \\
\left.\sum_{k=1}^{K_{e}^{(m)}} \sqrt{2 P_{k}} \mathbf{c}_{k}\left(t-\tau_{m j}\right)\right]
\end{array}
$$

where $\mathbf{h}_{m j}(t)=h_{m j}(t) \exp \left(j \varphi_{m j}\right)$ is the channel gain from satellite $m, \delta_{0}[n]$ denotes the Kronecker delta function, $P_{k}$ is the power transmitted to the $k$-th user, $\mathbf{c}_{k}(t)$ denote the spreading waveforms and $\tau_{m j}$, uniformly distributed in the signaling interval $T$, is the modulo- $T$ delay from satellite $m$. The transmitted power $P_{k}$ can be written as $P_{k}=P_{0} \eta_{k}$, where $P_{0}$ is the nominal power associated to a single user received at the satellite antenna beam center under unobstructed multipathfree conditions and $\eta_{k}$ denotes the power advance forced by the control scheme. We assume, without loss of generality, $\tau_{i}=0, \varphi_{i}=0$. In (1), the first term is the useful one, which is not zero only if the received signal $r_{m j}$ comes from the serving satellite, whereas the second one accounts for the MAI contribution. The number of terms in the related summation is expressed as the equivalent load $K_{e}^{(m)}$ which contributes to the interference coming from satellite $m$ (see [3] for a similar approach). Assuming the same average load for each carrier, beam and satellite, it results:

$$
K_{e}^{(m)} \triangleq E\left\{K_{I}^{(m)}[n]\right\} \cong \frac{K}{N_{c}} \rho \alpha \frac{\gamma_{0}-\zeta_{m}}{N_{b}}
$$

where $\alpha$ is the average value of the voice-activity factor, $\rho$ is the polarization isolation factor, $\gamma_{0}=\left[\int_{0}^{\theta_{b}} G[\theta] d \theta\right]^{-1}$ is the beam overlap factor out of the beam width $\theta_{b}$ and $\zeta_{m}$ is the global interference reduction term due to orthogonality, which turns out to depend on the number of users per satellite $K$. It should be noticed that the term $\gamma_{m} \triangleq \gamma_{0}-\zeta_{m}$ is the ratio of the total MAI coming from the whole $m$-th satellite to the interference that the user experiences only from its own spot beam (see [8] and references therein). For those satellites not engaged in the connection, namely for $m \neq i$, the received signal is totally unsynchronized: $\zeta_{m}=0$ and $\gamma_{m}$ reduces to $\gamma_{0}$. In these cases the equivalent number of users $K_{e}^{(m)}$ reaches its maximum value. As for the reference satellite $i$, no interference is experienced by the user if the satellite load is below the codebook limit, namely $\gamma_{i}=0$ for $K / N_{c} \leq 2 N$, being $N$ the spreading factor. When the satellite load increases over this limit, some non-orthogonal transmissions have to be superimposed on top of orthogonal multiplexing and $0<\gamma_{i}<\gamma_{0}$.

As $K$ becomes large, the MAI contribution of each satellite can be approximated as a zero-mean Gaussian random variable (rv); its power, normalized to the channel gain, $P_{I}^{(m)}$, can be written as:

$$
\begin{aligned}
P_{I}^{(m)} & \approx E_{0} \eta R_{u} K_{e}^{(m)}=E_{0} \eta R_{u} K \rho \alpha \frac{\gamma_{m}}{N_{b} N_{c}} \\
& =\left\{\begin{array}{lll}
I^{(s)} W & \text { for } & m=i \\
I^{(u s)} W & \text { for } & m \neq i
\end{array} \quad\right. \text { (synch. interf.) }
\end{aligned}
$$

being $E_{0}=P_{0} / R_{u}$ the nominal bit energy, $R_{u}$ the user data rate, $W$ the spreading bandwidth and $\eta=E\left\{\eta_{k}\right\}$ the average value of the power advantage required by the power control system. Notice that, in a full load condition, each MAI term can be written as a function of the total power $P \cong K E_{0} \eta R_{u} \alpha$ 
received from the satellite, namely:

$$
I^{(s / u s)} \cong \frac{P}{W} \frac{\gamma_{m} \rho}{N_{b} N_{c}} .
$$

In what follows, the latter expression will be used to compute the MAI contributions $I^{(s / u s)}$, directly in function of some key system design constraints, such as spacecraft power $(\propto$ $P)$, bandwidth $\left(W N_{c}\right)$ and number of beams.

\section{Link and Capacity Analysis}

In the following, a novel methodology for link and capacity analysis which takes into account satellite power constraints and BER-driven power control is described. The proposed approach is based on the calculation of the average power cost per user, namely the power factors $\eta_{j}$, conditioned to a certain propagation scenario. These values depend jointly on the value of the local shadowing attenuation and on the required target value $\Gamma$ of $E_{b} / N_{T}$ at the receiver output. This target value, on its turn, depends on the required error probability $P_{e}$ and on the residual statistic of $E_{b} / N_{T}$ after power control. In the uplink, the compensation of the shadowing attenuation can be disjoined by the control, on a statistical sense, of the fast fading component. In fact, given that the instantaneous $E_{b} / N_{T}$ is of the form: $\left(E_{b} / N_{T}\right)_{0} g \chi$, where $g \triangleq h^{2} / \chi$ is the local mean of the channel gain, the compensation of the long term variations eliminates the dependency from this local mean. A further margin is then required to counteract the effect of the residual fast fading term $\chi[5]$. In the downlink, the signal-to-noise ratio cannot be factored in a long term component and in a fast fading one. Hence, the computation of the power advance $\eta_{j}$, has to be performed by solving the following system:

$$
\left\{\begin{array}{l}
\left(\frac{E_{b}}{N_{T}}\right)^{(e s t)}=f_{\text {in }}^{\left(p_{\chi_{1}} \ldots p_{\chi_{M}}\right)}\left(g_{1} \ldots g_{M} ; \eta_{j}\right)=\Gamma \\
E\left\{Q\left(\frac{E_{b}}{N_{T}}\right)\right\}=f_{\text {out }}^{\left(p_{\chi_{1}} \ldots p_{\chi_{M}}\right)}\left(g_{1} \ldots g_{M} ; \Gamma\right)=P_{e}
\end{array}\right.
$$

In $\sqrt[5]{5},\left(E_{b} / N_{T}\right)^{(e s t)}$ denotes the measured signal-to-noise ratio, $g_{i}$ denotes the local mean of the channel gain related to the satellite $i, \Gamma$ and $P_{e}$, as previously indicated, denote the required target of $E_{b} / N_{T}$ at the output receiver and the required error probability, respectively, $p_{\chi_{m}}\left(\chi_{m}\right)$ is the probability density function (pdf) of the fast fading component $\chi_{m}$ and $Q(x)$ is the complementary cumulative Gaussian distribution. The average error probability is obtained by applying the statistical operator to the non compensated fast fading components. Notice that, in 5 , the first relationship models the inner loop logic, whereas the second one accounts for the outer loop effect. The measured $\left(E_{b} / N_{T}\right)$ expressions are obtained assuming a separate observation of signal and noise, namely: $\left(E_{b} / N_{T}\right)^{(e s t)}=E\left\{E_{b}\right\} / E\left\{N_{T}\right\}$, where the statistical operator is intended to filter out the fast fading component. It is easy to verify that the following holds, in case of SD:

$$
\left(E_{b} / N_{T}\right)^{(e s t)}=E_{0} \eta_{j} \frac{g_{i}}{N_{0}+I^{(s)} g_{i}+I^{(u s)} \sum_{m=1, m \neq i}^{M} g_{m}} .
$$

Hence, according to (5), the computation of each margin $\eta_{j}$ turns out to be conditioned to specific hypothesis about the channel state $\Theta=\Theta\left(g_{1} \ldots g_{M} ; p_{\chi_{1}} \ldots p_{\chi_{M}}\right)$, being $\Theta=\Theta^{(s)}$ with probability $p^{(s)}$, and $s=1 \ldots S$. Assuming the probabilities $p^{(s)}$ time invariant (large population hypothesis) $\eta_{j}$ can be modeled as a rv with probability mass function $p^{(s)}$ when $\eta_{j}=\eta^{(s)}$, being $\sum_{s=1}^{S} p^{(s)}=1$. As for the satellite channel, the two state propagation model is assumed [10] for each propagation environment: where the influence of the direct component cannot be neglected, the envelope of the received signal is modeled as a Rice process; otherwise (user shadowed from the satellite) the envelope is modeled as a Rayleigh-lognormal process. The propagation environment, on its turn, is classified into $L$ classes, basically depending on the urbanization degree and on the elevation angle $\psi$ experienced by the user. Hence, $g_{m}=1+1 / c_{l}$ if satellite $m$ is in LOS condition, being $c_{l}$ the Rice factor modeling the $l$-th propagation environment; otherwise, $g_{m}$ itself turns out to be a lognormal rv. As a matter of fact, this would lead to unlimited cardinality of the channel states set $\left\{\Theta^{(m)}\right\}$, so that the actual value of $g$ has been replaced by its average one $g^{(a v)}$, in order to access the model in its numerical form. The effect of this approximation has been found to be negligible in the overall dynamic range of the outer loop process. Hence, the long term attenuation $g_{i}$ can assume the following value:

$$
g_{i}= \begin{cases}10^{\frac{\mu_{l}}{10}+\left(\frac{\sigma_{l}}{10}\right)^{2} \frac{\ln (e)}{2}} & \text { if satellite } i \text { is shadowed } \\ 1+\frac{1}{c_{l}} & \text { otherwise }\end{cases}
$$

where $\mu_{l}$ and $\sigma_{l}^{2}$ denote mean and variance of the normal process which models the logarithmic expression of the long term fluctuation in the $l$-th environment. The residual fluctuations 
$\chi_{i}$, on its turn, is central or noncentral chi-square distributed provided that the satellite $i$ is shadowed or not, respectively.

\section{Numerical results}

Numerical results are derived for the voice service, according to the basic parameters of the $\mathrm{W}-\mathrm{CDMA}$ radio interface [7] [9]: $W=5 \mathrm{MHz}, R_{u}=8 \mathrm{kbps}, \alpha=0.5, N=128$, $P_{e}=10^{-3}, \rho=0.55$ (corresponding to a cross-polarization attenuation $A_{\rho}$ of $10 \mathrm{~dB}$ ) and $\gamma_{0}=2$. As for the system parameters, a 10 beams coverage, and a single carrier system has been assumed, namely: $N_{c}=1$ and $N_{b}=10$, whereas the the $\beta$ parameter, defined as $\beta \triangleq P /\left(N_{0} W\right)$, accounts for the spacecraft power. According to approximation (4), this parameter provides an estimate of the expected interference to noise spectral density ratio, as well, directly from the basic system parameters as beam shaping and available bandwidth, namely:

$$
\frac{I^{(s / u s)}}{N_{0}} \cong \beta \frac{\gamma_{m} \rho}{N_{b} N_{c}}
$$

A $\beta$ value of $20 \mathrm{~dB}$ in the $2 \mathrm{GHz}$ band, for instance, can be deemed typical of a Medium Earth Orbit (MEO) system, or a Low Earth Orbit (LEO) system with a moderate power payload (EIRP less than $30 \mathrm{dBW}$ ) [8]. The results are also based on the following characterization of the satellite system in terms of user classes: each user can be located in urban areas with probability $B_{u}$ or in open areas with probability $B_{o}=1-B_{u}$, so that 2 possible propagation scenarios can be envisaged, for each elevation $\psi$. A balanced distribution of users between the two classes is assumed, namely $B_{u}=B_{o}=0.5$, which is suitable to application scenarios where the satellite component accepts only the traffic overflow from the terrestrial networks. Urban and open areas are experienced as the worst and the best case, respectively. Let us denote by $A_{u / o}$ the shadowing probability in urban/open environment. The channel parameters in urban/open environment has been assumed according to the values reported in [10]. If we denote by $S^{(\psi)}$ the number of states, conditioned to a certain elevation angle $\psi$, a total number $S^{(\psi)}=4$ can be envisaged in case of single satellite reception, according to the urban/open and shadowing/unshadowing hypothesis. In figure 1 the excess power $\Delta \eta$ required by a shadowed user with respect to a non shadowed one is plotted versus the satellite load. As the number of users is under the codebook limit (256 in the shown case), the excess power equals the one required in uplink. As the interference increases and tends to dominate with respect the thermal noise, both signal and disturbances tend to be affected by the same attenuation and, hence, the instantaneous $E_{b} / N_{T}$ becomes more stable and tends to $E_{0} \eta_{j} / I^{(s)}$ for all users, thus reducing the differences in the power allocation. Significant insight about the effectiveness of a BERdriven power control, in terms of capacity gain, is provided by figures 2 and 3 . The gain is computed with respect to a pure SIR-based strategy, designed on a "worst-case" assumption. Figure 2 shows this gain for a single reception scheme, which ranges between $7 \%$ and $30 \%$ of capacity, depending on $\beta$ and on the elevation angle. As the $\beta$ value increases, the increasing weight of MAI makes the power distribution optimization among the active users more and more effective: the first raising of the gain curves, in their left side, is based on this rationale. On the other hand, when the MAI term predominates definitely over thermal noise (i.e., a further increase of the $\beta$ value), greater stability is expected in the signal-to-noise ratios (as previously observed), and the BER curves set $f_{\Theta}\left(E_{b} / N_{T}\right)$ tend to degenerate to a single curve $f\left(E_{b} / N_{T}\right)$. In this case, the optimum power control is roughly equivalent to the simpler SIR-based scheme, as indicated by the low values of the gain curves, in their right side. Greater sensitivity versus the power management criteria, and hence a major role of the outer loop process, can be clearly envisaged as the quality requirements increases: if the channel BER is reduced to $1 \cdot 10^{-2}$, a capacity gain up to $40 \%$ can be achieved with a BER-driven approach. As satellite diversity reduces the differences in the power allocation among the active users, a reduced capacity gain is expected, as it can be noticed in 3 . Nonetheless, for high values of $\beta$ (around $30 \mathrm{~dB}$ : LEO systems in the $2 \mathrm{GHz}$ band [8]), typically expected for the third generation mobile applications, the capacity gain is in the range $20 \%-30 \%$.

\section{An improved Power Management Strategy}

The above described analysis and results have been carried out, due to the fact that in satellite systems power is major concern. In fact, satellite payloads are power-limited and need to operate the on-board Power amplifier (PA) efficiently, i.e. close to their maximum power. However, the consequent PA 


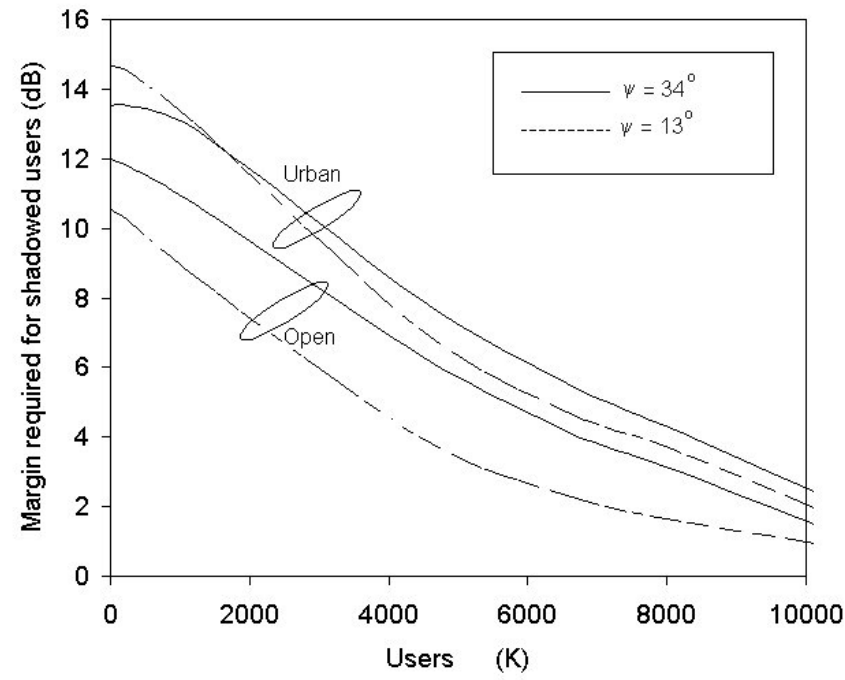

Fig. 1: Power margin versus satellite users.

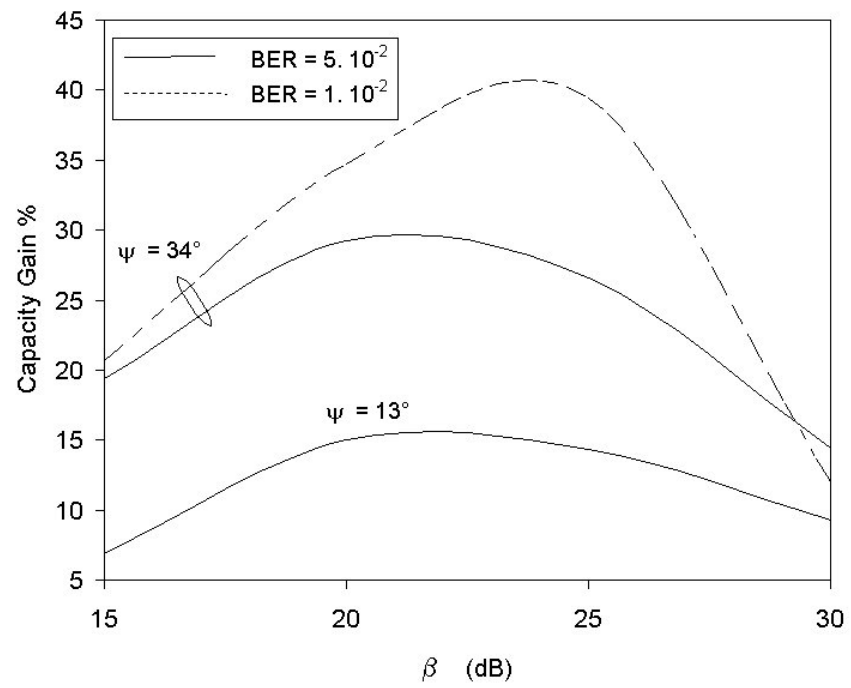

Fig. 2: Capacity gain of the BER-Driven power control with respect of the SIR-based power control in a single satellite system.

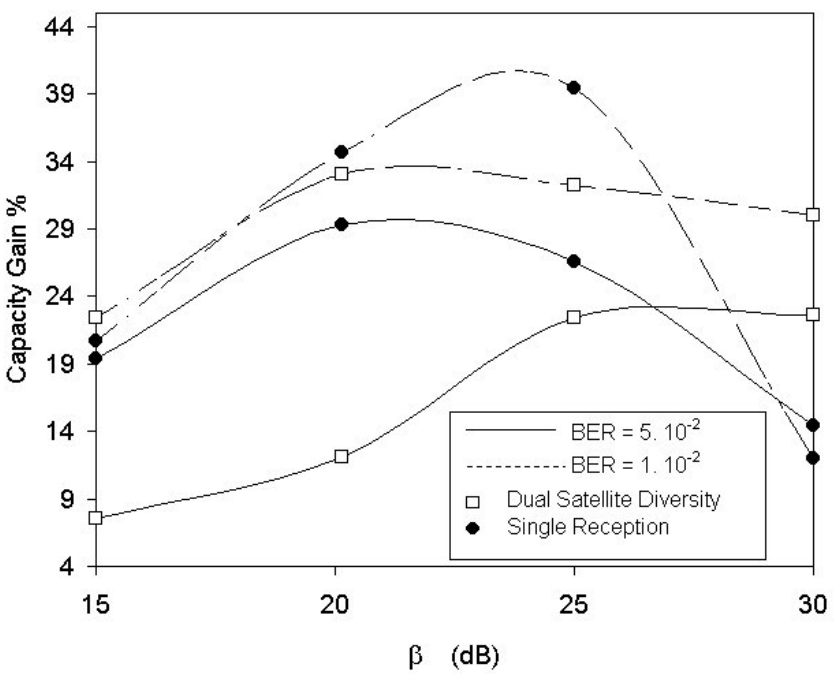

Fig. 3: Capacity gain of the BER-Driven power control with respect of the SIR-based power control in a dual satellite system. term which take into account all the factors reducing the interference contribution (activity factor, polarization isolation, orthogonality, and so on) and $N_{0}$ is the power spectral density factor of the thermal noise. The received power is $P_{R}=P A$ 
where $A$ is the total attenuation, the SNR can be written:

$$
S N R=\frac{P A}{N_{0}+\delta I A}
$$

All the transmitted powers are now reduced of a factor denoted with $\Delta(<1)$. Also, reducing the total transmitted power a reduction of the loss of orthogonality can be achieved. This reduction leads to a reduction of the interference contribution of $\Delta_{P A}(<1)$. It is straighforward to show that the same SNR can be achieved reducing the transmitted power levels of $\Delta$ such that:

$$
\Delta=\frac{N_{0}}{N_{0}+\delta I A\left(1-\Delta_{P A}\right)} .
$$

Therefore, it should be suitable a forced system rearrangement which allows to find a new balance condition corresponding to a lower total transmitted power. Periodically, the system could reduce of a fixed quantity all transmitted power levels waiting that, through the normal power control mechanisms, the system reaches a new balance condition.

\section{Conclusions and Perspectives}

We have analyzed the performance of a CDMA-based mobile satellite system with a BER-Driven power control scheme. The analysis has been developed according to a novel approach specifically intended for the power-limited satellite-tomobile link with multi satellite reception. This proposed approach is based on the characterization, on a statistical sense, of the outer loop process which affects the SIR target value, according to actual BER estimation. A strong sensitivity of capacity versus the power management criteria has been found: the gain obtainable with respect to a pure SIR-based strategy can reach the $40 \%$ of the total capacity in a single reception scheme, whereas it reduces to $20 \%-30 \%$ with satellite diversity. Hence, due to the twofold effect of power control in MAI reduction (CDMA- induced effect) and in the optimization of power distribution among the active users (satellite-induced effect), the use of a BER-driven approach appears to be a valuable solution for power management policy in the future mobile satellite systems. The described analysis can be improved taking into account the uncertainty in the SNR and BER estimates through a proper modeling. Furthermore, as future integrated systems will have to support innovative services with IP technology, power management strategies for packet-switched communications have to be considered and analyzed.
The usage of the analysis methodology for an accurate evaluation of the proposed forced system rearrangement, in terms of capacity or power saving, taking properly into account the effects of the nonlinearity is presently an on-going activity of the Authors' work.

\section{References}

[1] E. Dahlman, P. Beming, J. Knutsson, F. Ovesj, M. Persson, C. Roobol, WCDMA-The Radio Interface for Future Mobile Multimedia Communications, IEEE Transactions on Vehic. Tech., Vol. 47, No. 4, Nov. 1998, pp. 1105-1117.

[2] T. Ojanpera, R. Prasad, Wideband CDMA for Third Generation Mobile Communications, Arthech House, 1998.

[3] R. De Gaudenzi, F. Giannetti, DS-CDMA Satellite Diversity Reception for Personal Satellite Communication: Satellite-to-Mobile Link Performance Analysis, IEEE Transactions on Vehic. Tech., Vol. 47, No. 2, May 1998, pp. 658-672.

[4] B. R. Vojcic, L. B. Milstein, R. L. Pickholtz, Downlink DS-CDMA Performance Over a Mobile Satellite Channel, IEEE Transactions on Vehic. Tech., Vol. 45, No. 3, Aug. 1996, pp. 551-558.

[5] B. R. Vojcic, L. B. Milstein, R. L. Pickholtz, Total Capacity in a Shared CDMA LEOS Environment, IEEE J. Select. Areas Commun., Vol. 13, No. 2, Feb. 1995, pp. 232-244.

[6] B. R. Vojcic, L. B. Milstein, R. L. Pickholtz, Power Control Versus Capacity of a CDMA System Operating Over a Low Earth Orbiting Satellite Link, Proc. Communication Theory Mini-Conference Held in Conjunction with Globecom '93, pp.40-44.

[7] S. De Fina, On the Capacity of UMTS Satellite Component, Mobile and Personal Satellite Communications (M. Ruggieri, Ed.), Springer Verlang, 1999, chapter 3, pp. 254-261.

[8] E. Cianca, S. De Fina, M. Ruggieri, Spectral and Power Efficiency of the UMTS Satellite Component, Proc. IEEE Veh. Tech. Conference, May 16-20, 1999, Huston, Texas, pp. 1747-1751.

[9] Tdoc SMG2 258/98 PART B, UTRA Physical Layer Description FDD parts, v0.3, 1998-05-29.

[10] E. Lutz, D. Cygan, M. Dippold, F. Dolainsky, W. Papke, The Land Mobile Satellite Communication Channel-Recording, Statistics, and Channel Model, IEEE Transactions on Vehic. Tech., Vol. 40, No. 2, May 1991 , pp. 375-385. 